\title{
A Fade-Level Skew-Laplace Signal Strength Model for Device-Free Localization with Wireless Networks
}

\author{
Joey Wilson and Neal Patwari
}

\begin{abstract}
Device-free localization (DFL) is the estimation of the position of a person or object that does not carry any electronic device or tag. Existing model-based methods for DFL from RSS measurements are unable to locate stationary people in heavily obstructed environments. This paper introduces measurement-based statistical models that can be used to estimate the locations of both moving and stationary people using received signal strength (RSS) measurements in wireless networks. A key observation is that the statistics of RSS during human motion are strongly dependent on the RSS "fade level" during no motion. We define fade level and demonstrate, using extensive experimental data, that changes in signal strength measurements due to human motion can be modeled by the skew-Laplace distribution, with parameters dependent on the position of person and the fade level. Using the fade-level skewLaplace model, we apply a particle filter to experimentally estimate the location of moving and stationary people in very different environments without changing the model parameters. We also show the ability to track more than one person with the model.
\end{abstract}

Index Terms-Device-free localization, wireless networks, RF sensors, tracking, through-wall surveillance.

\section{INTRODUCTION}

$\mathrm{K}$ NOWING the location of people is extremely valuable and useful. Global navigation satellite systems, radio frequency identification (RFID), and real-time location systems (RTLSs) have proven their value for locating people and assets with an attached device. Device-free localization (DFL) is the practice of locating people ${ }^{1}$ or objects when no tag or device is attached to the entity being located. DFL technologies are useful in applications where the people being tracked cannot be expected to cooperate with the system. This may be the case because they are intentionally evading the system, because they are physically unable, or because they do not want to be inconvenienced by wearing a tag. In this paper, we investigate a statistical inversion method for DFL in narrowband RF sensor networks, and show its effectiveness in tracking both moving and stationary objects located behind walls.

Various sensor technologies can be used for the purposes of DFL [1], as discussed in Section 4. In this paper, we are particularly interested in DFL systems which use received signal strength (RSS) measurements (RSS-DFL) because RSS can be measured with a variety of widely deployed and inexpensive wireless devices. RSS-DFL can locate motion through building walls [2], in dark or smoke-filled environments, and are not as invasive of privacy as video camera surveillance.

1. In this paper, we use the word "people" generally to refer to either people or objects that are to be located and tracked.

- The authors are with the University of Utah, Room 3280 MEB, 50 South Campus Drive, Salt Lake City, Utah 84108.

E-mail: joey@xandem.com, npatwari@ece.utah.edu.

Manuscript received 15 Sept. 2010; revised 2 Mar. 2011; accepted 8 Apr. 2011; published online 29 Apr. 2011.

For information on obtaining reprints of this article, please send e-mail to: tmc@computer.org, and reference IEEECS Log Number TMC-2010-09-0434. Digital Object Identifier no. 10.1109/TMC.2011.102.
RSS-DFL systems have, to this point, significant limitations. Imaging-based RSS-DFL systems first estimate attenuation or motion image and then estimate the person's coordinate from that image [2], and information can be lost in the two-step process. In particular, variance-based radio tomographic imaging (VRTI) cannot be used to locate a stationary (or very slow-moving) person. Fingerprint-based RSS-DFL systems require extensive calibration measurements [3], [4]. To date, direct coordinate estimators in RSSDFL do not benefit from accurate statistical models for the RSS measurements [5], [6].

To provide a means to address these significant limitations, in this paper, we present a new statistical inversion method for RSS-DFL in wireless networks. The new model allows for direct estimation of a person's position, without the need to use radio tomographic images as an intermediate information layer.

This statistical inversion method is enabled by a new RSS model presented in this paper for temporal fading on static links. Significant statistical models exist for small-scale fading, but this model represents an advance on two levels. First, the model presented is a function of the current position of a person-whether or not the person is now close to the link. Second, the model presented is a function of the fade level, that is, a quantification of the narrowband fading experienced on the static link prior to the person's appearance in the environment. Fade level is a measurable quantity in RSS-DFL. The new model takes advantage of the uniqueness of each link in the RF sensor network, as quantified by the fade level, rather than assuming each link behaves identically when people are located near a link. We show that links experience drastically different behavior as a function of the fade level.

Our model is based on extensive measurements conducted in two very different environments in which DFL 


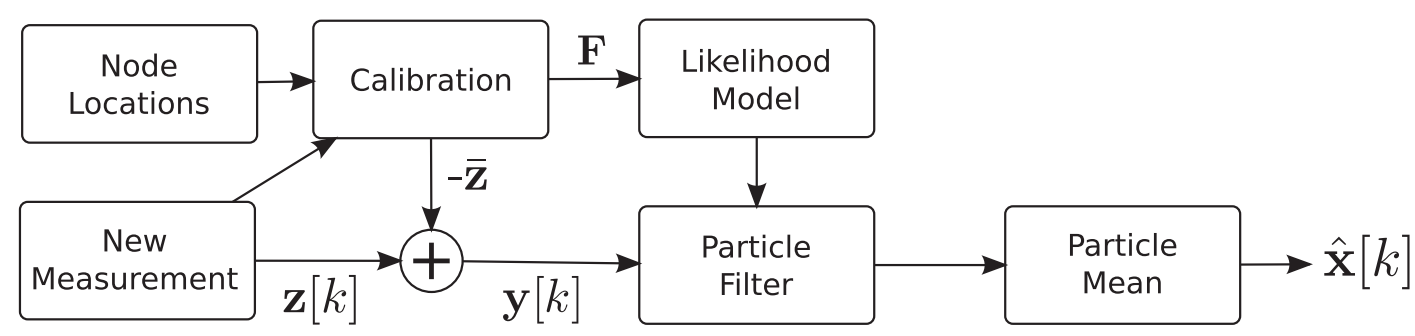

Fig. 1. A flowchart describing the statistical inversion process for device-free localization in wireless networks.

systems are expected to operate. We find that the temporal variation of RSS is well modeled with the skew-Laplace distribution. Our measurements quantify the relation between the parameters of the skew-Laplace distribution to a person's location and a link's fade level.

Finally, we demonstrate the application of the fade-level skew-Laplace model in a statistical inversion method in a real-world system. We demonstrate that the method is able to locate motionless people through external building walls, which had not been demonstrated in previous work. We show that moving people can be tracked with less than 1 meter error in our experiments.

The statistical approach allows us to address some key limitations of previous RSS-DFL systems. Since the new method does not rely on manual site-specific measurements, it can be deployed at multiple sites without the need for offline training. All that is needed is a short calibration period (a few seconds) where the network is assumed to be free from moving objects to determine the means of each link. The training, in essence, has already been performed in the modeling of the statistics. Furthermore, the new method does not require a specific network location geometry or regularity in the environment.

The statistical inversion process is described in detail in Section 2. An overview is provided in Fig. 1 . Let $M$ be the total number of links in the network. A $M \times 1$ raw RSS measurement vector $\mathbf{z}[k]$ for each link in the network is received at time $k$ at a base station processing unit. Raw calibration measurements are collected during a period during which the network is assumed vacant, or over a longterm history. These calibration measurements are combined with knowledge about the node locations to determine the means $\overline{\mathbf{z}}$ and fade-levels $\mathbf{F}$. During operation, the link means are subtracted from the incoming measurement to determine the change in signal strength $\mathbf{y}[k]=\mathbf{z}[k]-\overline{\mathbf{z}}$.

The fade-level calibration information is used to determine the statistical likelihood model based on the skewLaplace distribution, as discussed in Section 2. The likelihood model provides the basis for particle filtering, a nonlinear and non-Gaussian filter for recursive estimation, which is used to infer location results $\hat{\mathbf{x}}[k]$.

\section{Statistical Modeling}

\subsection{Overview}

In general, a statistical likelihood model represents the noisy translation from a state space to a measurement space (see Fig. 2). Given a particular state, a certain distribution of measurements will result. This can be thought of as a forward process, defined by likelihood distribution $P(Y \mid X)$, where $X$ is the state to be estimated, and $Y$ is received or measured data. The inverse problem, therefore, involves taking measured data and estimating the distribution of the state. The state likelihood is defined by the posterior distribution $P(X \mid Y)$, found by applying Bayes' theorem

$$
P(X \mid Y)=\frac{P(Y \mid X) P(X)}{P(Y)} .
$$

For DFL applications, the state-space $X$ is the coordinates of device-free entities within a wireless network, and the measurements $Y$ are RSS values of each link in the network. We take the RSS measurements and infer the position of the people by inverting the statistical model through the posterior distribution.

The likelihood function $P(Y \mid X)$, and the a priori, knowledge of the state described in $P(X)$, describes the statistical model that can be used to invert the problem. We are, therefore, interested in knowing how the position of people affects the resulting RSS measurements, and how those statistics change for different positions of the people. We expect a person standing on the line-of-sight (LOS) of a link to cause significant changes to the RSS measurements, while a person at a distant position away from the LOS will not. The statistics for each link-person geometry are modeled in the likelihood functions.

The a priori, information $P(X)$ can be used to incorporate known information about the people. Since people must move with finite velocity, this information allows an inversion algorithm to more accurately estimate positions over time. If movements are constrained by walls or other obstacles, the probability of transitions through those obstacles can be set to zero, thus improving accuracy and efficiency. In our experiments in Section 3, we constrain the domain by only searching over the interior area of the home.

\subsection{Measurement Collection}

To form a likelihood model, an experimental RF sensor network is deployed to capture RSS measurements. The

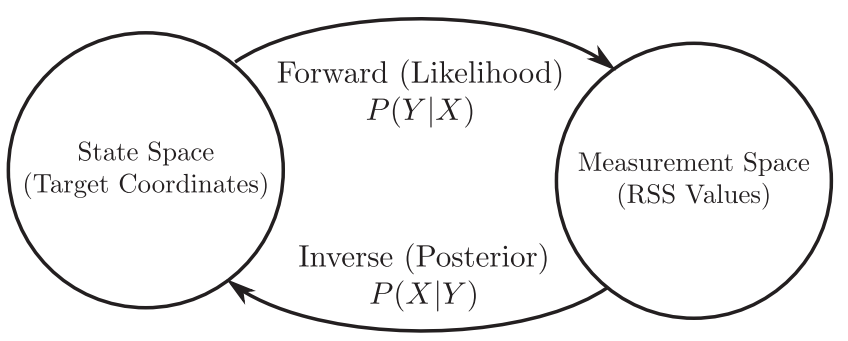

Fig. 2. An illustration showing the role of likelihood and posterior distributions for statistical inversion. 


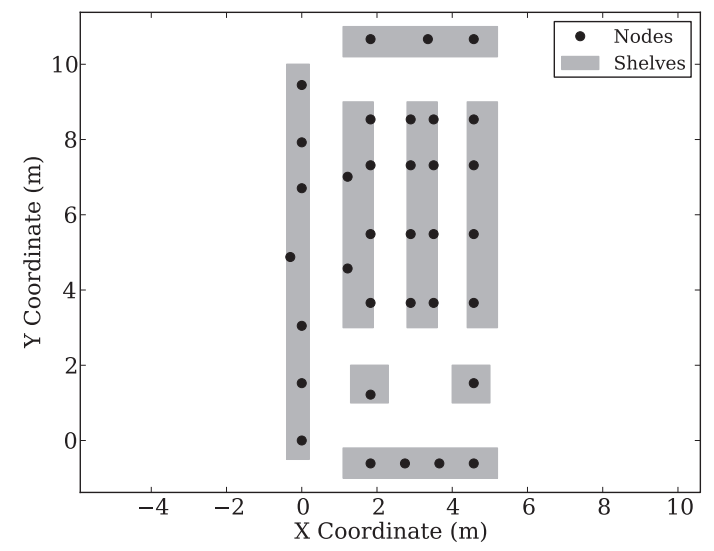

Fig. 3. The layout of a 34-node bookstore deployment.

network nodes consist of 34 TelosB nodes from Crossbow, each utilizing the IEEE 802.15.4 protocol in the $2.4 \mathrm{GHz}$ frequency band.

A token passing protocol called Spin, available at [7], is used to prevent wireless packet collisions while maintaining low data collection latency. Each node is assigned an ID number and programmed with a known order of transmission. When a node transmits, each node that receives the transmission examines the sender identification number. The receiving nodes check to see if it is their turn to transmit, and if not, they wait for the next node to transmit. If the next node does not transmit, or the packet is corrupted, a timeout causes each receiver to move to the next node in the schedule so that the cycle is not halted. A base-station node that receives all broadcasts is used to gather signal strength information and pass it to a laptop computer for processing.

The experimental network is deployed in two areas, one throughout the aisles of the University of Utah campus bookstore, and one around the outer perimeter of part of a house. Both environments are rich in multipath, and the natural state of each of the environments was not modified for our experiments. The data are collected from multiple deployments so that the statistical analysis of changes in signal strength remain untied to a particular environment.

In the bookstore deployment, nodes are placed on shelves and stands at approximately human waist level. Some links cross through multiple aisles, and some remain in direct LOS. There are no walls that separate the nodes in the network, but furniture such as book shelves, books, tables, promotional displays, and other products provided many obstructions. The layout of this deployment is shown in Fig. 3.

In the house perimeter deployment, the network is deployed in an area outside of a portion of a typical singlefamily house. Three of the walls are external (constructed of wood, insulation, and cement board siding), and one is located on the interior of the house. The interior wall is constructed of brick, wood, and plaster-before an addition to the house, it was also an external wall. In this deployment, the nodes are placed in a rectangular perimeter, as depicted in Fig. 4. It is neither possible, nor necessary, to place the nodes in a uniform spacing due to building and property obstacles. Eight of the nodes are

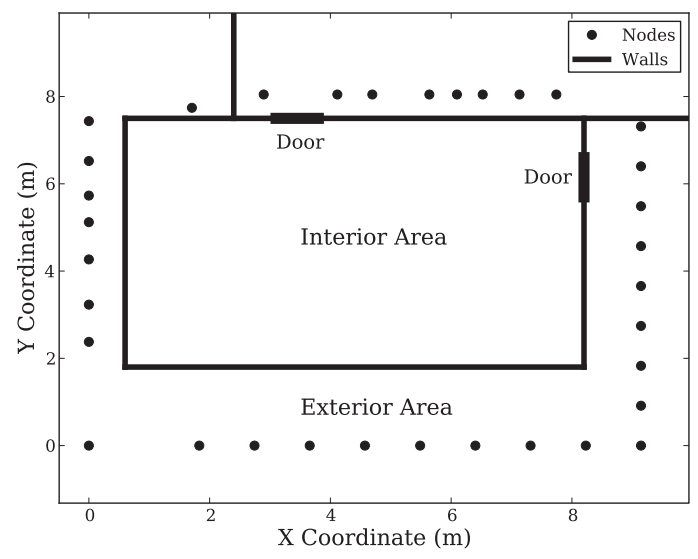

Fig. 4. The layout of a 34-node through-wall wireless network deployment.

placed on the inside of the building, but on the other side of the brick interior wall.

RSS data are gathered as humans walk near and through the networks. The location of each person is carefully tracked by having each person step in a predetermined path defined by markers placed on the ground. To keep each person moving at a constant velocity, an audible metronome is played over a speaker, allowing each person to step to the next marking at the correct time. Using this technique, millions of RSS measurements are gathered, each measurement synchronized with knowledge of the actual positions of each person.

Since our likelihood models are based on changes in signal strength, a calibration process is used for each deployment. During calibration, RSS measurements for each link are taken while the network area is vacant of people. The calibration mean for link $l$, which we denote $\bar{z}_{l}$, is set to the average of RSS measurements during this calibration period.

\subsection{Fading Information}

People moving near a wireless link will cause changes in RSS. This temporal variation is different from small-scale or frequency selective fading that occurs due to relative motion between the transmitter and receiver in multipath environments. During motion of a transmitter or receiver, the phase of all multipath components change as the path length changes. In contrast, the presence of the person near the wireless link affects only a subset of multipath components in the link [2], [8].

When the channel is predominantly LOS, such as in an open outdoor area, then a human crossing the LOS will generally cause a drop in signal strength due to shadowing of the LOS path. This phenomenon has been applied to image the attenuation of humans within a wireless network [9].

When an environment is rich in multipath and heavily obstructed, the presence of a human on the LOS of a link causes less predictable changes in RSS. In different cases, the link RSS may decrease, remain unchanged, or increase. To take advantage of temporal changes in RSS on obstructed links, regardless of the direction of the change, variance-based radio tomographic imaging [2] may be applied. The key weakness of VRTI, however, is that people 


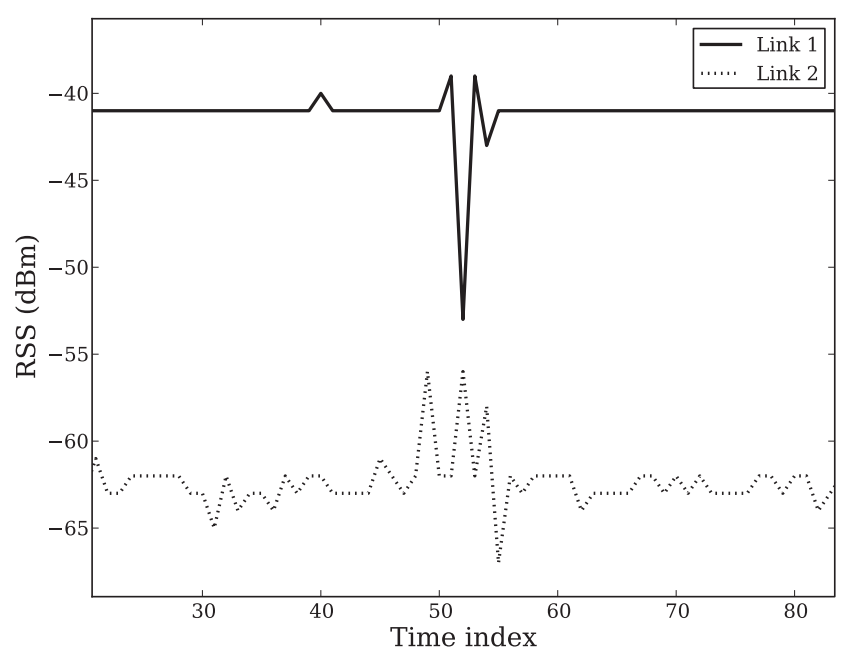

Fig. 5. An example of how the RSS statistics for two links of equal distance and in the same environment are drastically affected by the fade level. Here, a human crosses through the LOS at $t=52$. Link 1 is in an "antifade" while Link 2 is in a deep fade.

must remain moving in order to result in measurable temporal variation, and thus be able to be tracked. Stationary people, or those that move very slowly, will not be imaged.

Note that the steady state of narrowband fading on a link, when there is no person near the link, plays an important role in the statistics of the changes caused when there is a person near the link. We define and describe two extremes of the narrowband fading experienced by a static link.

- Deep fade. A link that experiences deep multipath fading (i.e., destructive multipath interference) without the person present is more likely to experience a high variance of RSS when a person enters the area. Further, this link will, on average, measure an increase in RSS due to the person's presence.

- Antifade. On the other hand, a link that experiences constructive multipath interference when the person is not present, will vary significantly less due to the person's presence, and further, will measure, on average, a decrease in RSS. We use the term "antifade" to be the opposite of the common term "deep fade."

These observations about temporal fading due to human presence has been verified by simulation [10].

To illustrate this phenomenon from our measurement observations, an example of how RSS varies over time for two links of equivalent distance is shown in Fig. 5. Since the path lengths are the same for the two links, one can observe that the fade-level difference is $20 \mathrm{~dB}$. We can consider Link 1 to be in an antifade and Link 2 to be in a deep fade. As a human walks through the LOS path of the two links, the RSS changes. In the case where the link is in an antifade, very little variance is experienced when the person is not directly between the nodes. When the person crosses, the RSS drops significantly since the link was previously experiencing constructive multipath interference. Any disruption to the phases or amplitudes of the multipath would, therefore, bring the power down. In the deeply faded link, the opposite is true; any disruption to the multipath components of the link tends to bring the power up. In Link 2 the RSS generally increases while the human walks through the LOS, as seen in the figure.

We build a statistical model for changes in RSS due to human presence as a function of the narrowband fading level in Section 2.4.

\subsection{Quantification of Fade Level}

We now quantify the amount of fading occurring on a static link by defining a "fade level." In a wireless channel, the ensemble mean $P(d)(\mathrm{dB})$ measured by the receiver is dependent on the distance $d$ from the transmitter

$$
P(d)=P_{T}-\Pi_{0}-10 n_{p} \log _{10} \frac{d}{\Delta_{0}},
$$

where $P_{T}$ is the transmitted power in $\mathrm{dBm}, n_{p}$ is the path loss parameter, and $\Pi_{0}$ is the loss measured at a short reference distance $\Delta_{0}$ from the transmitter. For more information on the derivation of this equation and its associated parameters, see [11].

In multipath environments, fading will cause a significant deviation from the ensemble mean in (2). We quantify the fade level as the difference between the path loss prediction and the calibration mean $\bar{z}_{l}$ in $\mathrm{dB}$ for link $l$

$$
F_{l}=\bar{z}_{l}-P\left(d_{l}\right),
$$

where $F_{l}$ is the $l$ th element of $\mathbf{F}$ and $d_{l}$ is the length of link $l$. Assuming the locations of each node are known or estimated in a wireless network, it is simple to calculate the fade level for each link. Calibration provides the link mean $\bar{z}_{l}$, and the path loss model is applied to determine $P\left(d_{l}\right)$, based on a known path loss parameter and reference powers.

The path loss parameter can be estimated using calibration data. The average signal strength for each link $\bar{z}_{l}$ is recorded during the calibration phase, and the distance of each link is known since all node positions are known. A least-squares linear fit is used to determine the parameters of (2) that best fit the calibration mean data.

\subsection{Measurement and Modeling Results}

No current model exists for the statistics of temporal variation on a wireless link as a function of the static fade level. To obtain such a model, we bin each RSS measurement according to its known fade level found during calibration. Additionally, we separate RSS measurements for when a person is located on the direct LOS path, versus no person located on the direct LOS path. We then examine histograms for each bin of fade level and LOS condition. The distribution of RSS measurements when a person is on the LOS for low fade levels is found to have a heavier tail in the positive direction, while the distribution for high fade levels has a negative skew. Histograms of the data are shown in Fig. 6. For fading levels of $-15 \mathrm{dBm}$ and less, the decay rate on the positive side of the skew-Laplacian is much lower when a person is standing on the LOS path than when a person is not on the LOS. On the other hand, when the fade level is greater than $10 \mathrm{dBm}$, we see that tail on the negative side of the distribution is longer. When the person is not on the LOS path of the link, the variance of the 

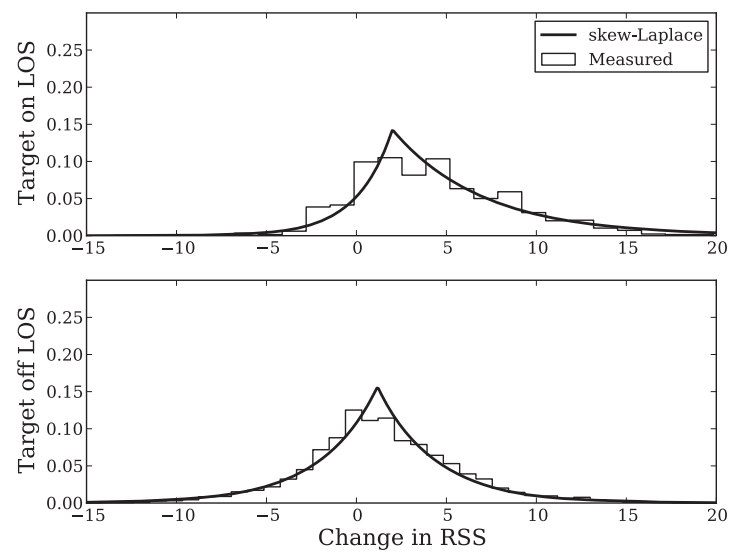

(a) Deep fade
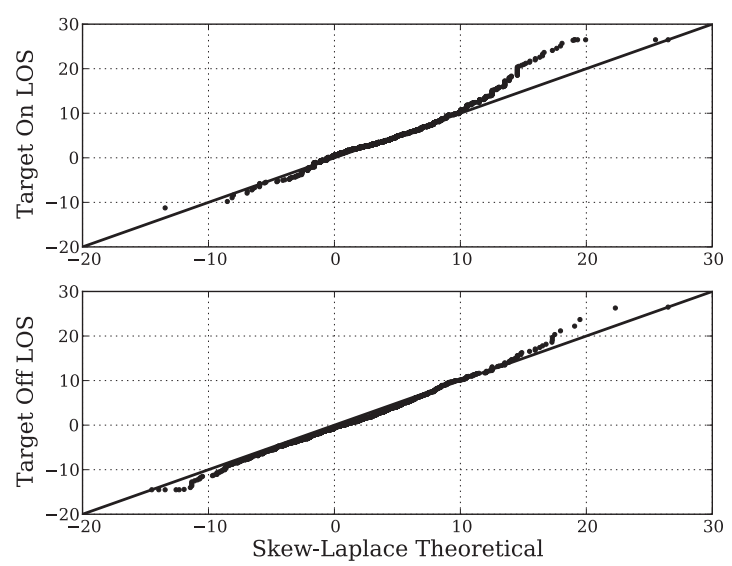

(c) Deep-fade
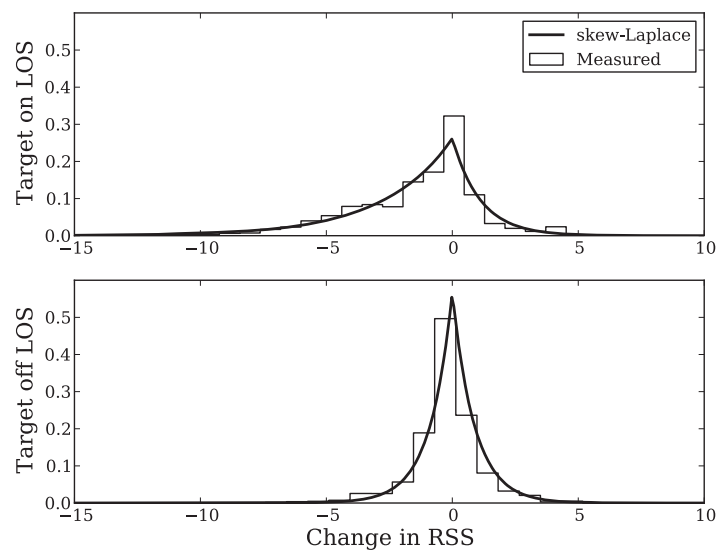

(b) Anti-fade
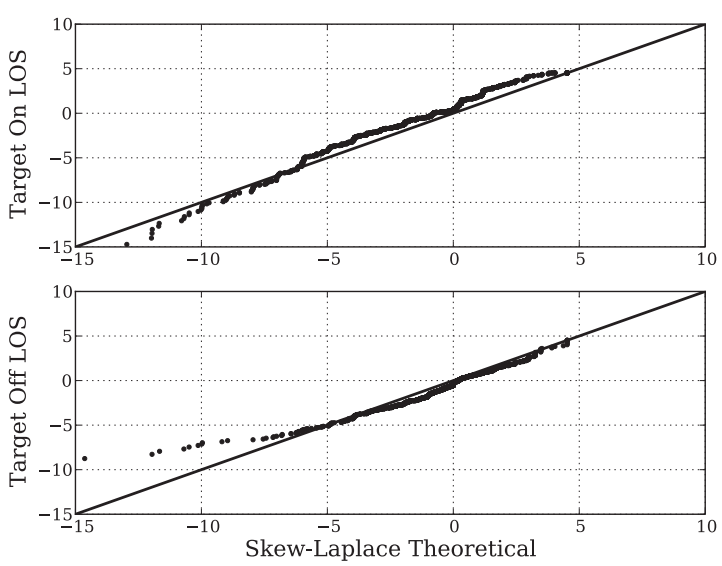

(d) Anti-fade

Fig. 6. RSS measurement distributions for on/off LOS person positions. The histograms for links experiencing deep fades (fade level less than $-15 \mathrm{dBm}$ ) is shown in (a) and those experiencing antifades (fade level greater than $10 \mathrm{dBm}$ ) in (b). Quantile-Quantile plots for both cases are shown in (c) and (d), where the $x$-axis represents the theoretical skew-Laplace quantile, and the $y$-axis represents the measured quantiles.

distribution is significantly lower. The evidence provided by the data in these histograms supports our heuristic argument that links already in a deep fade should rise in power when disturbed, and links already in an antifade should decrease in power when disturbed.

With the understanding that the skew of the RSS distribution is dependent on the static fade level, a nonsymmetric probability density function with both positive and negative support is desired. The skew-Laplace distribution meets these criteria, and fits the measurements well as seen in the quantile-quantile plots of Fig. 6. The distribution is defined as

$$
f(s ; a, b, \psi)=\frac{a b}{a+b} \begin{cases}e^{-a(\psi-s)}, & \text { if } \mathrm{s} \leq \psi, \\ e^{-b(s-\psi)}, & \text { if } \mathrm{s}>\psi,\end{cases}
$$

where $a$ and $b$ represent one-sided decay rates of the distribution for values less than or greater to the mode $\psi$. For the purposes of DFL, the values of each parameter of the skew-Laplace distribution are dependent on the fading level of the static link and the position of the person with respect to the link.

The RSS distributions shown in Fig. 6 represent the two extreme fading cases. When a link is neither in a deep fade nor an antifade, the parameters of the distribution will fall between those of the extremes. In other words, the parameters of the likelihood model are dependent on the value of fade level. These parameters are approximately linear with the fade level, and we use the least-squares criteria to determine the line of best fit. The linear fit equations are presented in Table 1.

The mode parameter $\psi$ for varying fade levels is shown in Fig. 7a. We see that when the person is off the LOS path, the mode parameter is near zero for all values of the fade level. When the person is located on the LOS path, a piecewise-linear function can be used to approximate the parameter for a given fade level.

The decay parameters for varying fade levels are shown in Figs. $7 \mathrm{~b}$ and 7c. All parameters can be approximated with piecewise-linear functions of the fade level. We see that as

TABLE 1

Linear Parameter Fitting for the Fade-Level Skew-Laplace Likelihood Model

\begin{tabular}{|c|c|c|c|}
\hline & $\psi$ & $a$ & $b$ \\
\hline LOS $(F \leq-6)$ & $-3.85-.631 F$ & $.350+.004 F$ & $.607+.028 F$ \\
\hline LOS $(F>-6)$ & 0.0 & $.350+.004 F$ & $.607+.028 F$ \\
\hline Off LOS & 0.0 & $.726+.019 F$ & $.902+.040 F$ \\
\hline
\end{tabular}




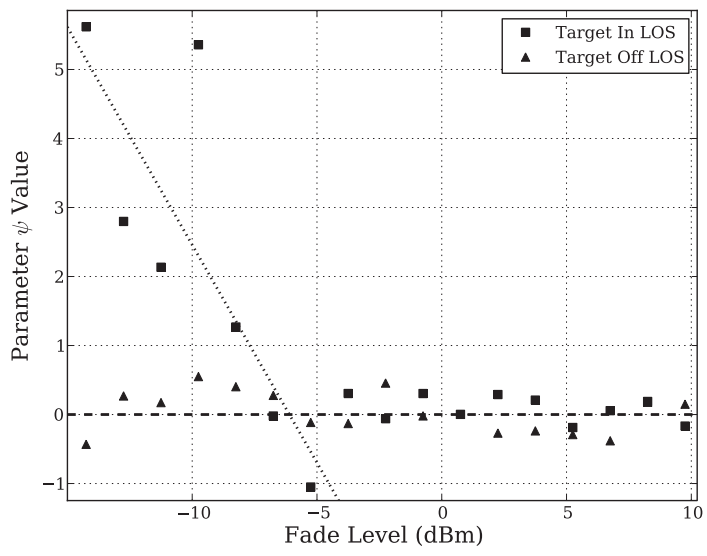

(a) Mode Parameters

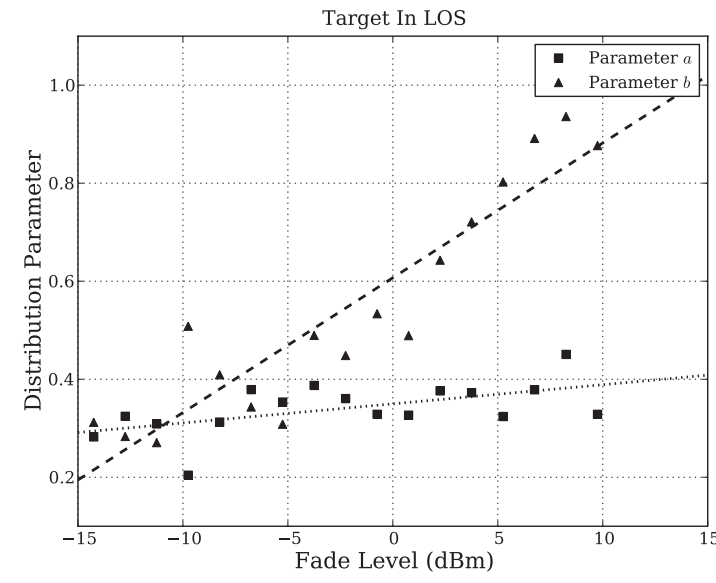

(b) Decays for person in LOS

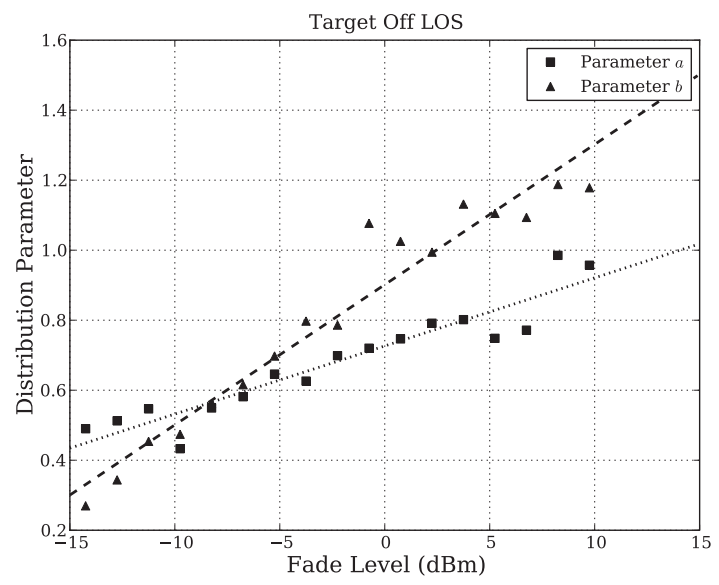

(c) Decays for person off LOS

Fig. 7. Parameter fitting for the fade-level skew-Laplace likelihood functions over a range of fade levels in dBm. Mode parameters are shown in (a). Decay parameters when the person is located on the LOS path of the link are shown in (b). Decay parameters when the person is off the LOS path are shown in (c).

the fade level increases, the decay parameters increase for both the on and off LOS cases. In other words, links that experience a deep fade have higher variance than those that experience an antifade.

To summarize the model, the distribution of RSS measurements is dependent on the existence of a person on the LOS path, and on the static fade level of the link. The values for each of the different cases are presented in Table 1. The fade level, as discussed previously, can be computed by deploying a network before any people have entered an area, or by processing measurements over time.

\section{Applying the Model: Tracking With PARTICLE FILTERS}

\subsection{Particle Filtering Algorithm}

There are many frameworks for estimating a posterior distribution using likelihood models. Kalman filtering, in its multiple forms, is by far the most common of these algorithms. For our application, the particle filter is an attractive form of posterior estimation, and a simple and brief outline of the particle filter applied in this paper is provided here. The derivation, theory, and variants of the particle filter will not be covered, as this information is widely available in the literature [12], [13], [14], [15].

There are a number of reasons why particle filtering is attractive for DFL in RF sensor networks. First, particle filters do not make any assumptions on linearity of the measurement process or the dynamics of the state being estimated. Since our likelihood models are dependent on the existence of a person on the LOS path of each link, this is an important flexibility. Furthermore, nonlinear models for person movement can be incorporated directly into the particle framework.

Second, unlike the Kalman filter, the particle filter does not require the likelihood distributions to be Gaussian. This is extremely important for applying our likelihood functions, as they are well modeled as skew-Laplacian. Assuming Gaussian distributions would be suboptimal, and may introduce significant tracking error.

Finally, the particle filter is attractive for real-time processing since incoming measurements can be used to update the posterior estimation without storing a history of previous measurements. As new measurements arrive, the algorithm recursively predicts and updates its estimation in a manner similar to that of the Kalman filter. 
The use of a particle filter for DFL is not without disadvantages. The primary weakness of particle filters is the computational complexity required to run the algorithm. The particle filter naturally relies on a high number of particles to achieve accurate results, at the expense of computational resources. There are many forms of the particle filter, including the auxiliary particle filter [13] and the unscented particle filter [14], which aim to increase efficiency and accuracy.

In this work, each particle represents a particular hypothesized location coordinate of a person. Let $\mathbf{x}[k]$ be the true location of the person at time $k$, and let the set $\left\{\tilde{\mathbf{x}}^{i}[k]\right\}_{i}$ be the set of particles that represent hypotheses of person position. Let the set $\left\{\tilde{w}^{i}[k]\right\}_{i}$ be the weights of each particle at time $k$, let $\mathbf{y}[k]$ be the current difference in RSS measurements for each link from the calibration data, and let $\hat{\mathbf{x}}[k]$ be the person location estimate. We use the following sampling-importance-resampling (SIR) [12] particle filter to perform our experiments.

1. Measure. Receive new measurement vector $\mathbf{z}[k]$ from each link in the network, then subtract the calibration mean $\overline{\mathbf{z}}$ to obtain $\mathbf{y}[k]$. Note that it is not necessary to process every link measurement at each period; a subset of measurements can be used.

2. Weight update. For each particle $\tilde{\mathbf{x}}^{i}[k]$ and each link RSS measurement, use the measurement vector $\mathbf{y}[k]$ to determine the updated weights.

- Determine skew-Laplace parameters $a_{l}, b_{l}$, and $\Psi_{l}$ for each link given the current particle $\tilde{\mathbf{x}}^{i}[k]$ using Table 1 . These are stored in $M \times 1$ vectors $\mathbf{a}, \mathbf{b}$, and $\Psi$.

- For each link, determine $p\left(y_{l}[k] \mid \tilde{\mathbf{x}}^{i}[k]\right)$ using the fade-level skew-Laplace likelihood model. Thus,

$$
p\left(y_{l}[k] \mid \tilde{\mathbf{x}}^{i}[k]\right)=f\left(y_{l} ; a_{l}, b_{l}, \psi_{l}\right),
$$

where $y_{l}[k]$ is the $l$ th element of $\mathbf{y}[k]$, and $a_{l}, b_{l}$, and $\psi_{l}$ are the $l$ th elements of each parameter vector determined in the previous step. Update weights with

$$
w^{i}[k]=w^{i}[k-1] p\left(y_{l}[k] \mid \tilde{\mathbf{x}}^{i}[k]\right),
$$

and normalize with

$$
w^{i}[k]=w^{i}[k] / \sum_{j} w^{j}[k] .
$$

3. Resample. Particles with heavy weights are reproduced, particles with very low weights are eliminated. We use the algorithm described in [12] to perform this task.

4. Move the particles. Apply a Markov transition kernel to each particle. In our experiments, we use the Metropolis-Hastings algorithm [16].

5. Estimate. Average the particles to obtain the mean of the posterior distribution as the current state estimate.

In this algorithm, we assume that the particle filter proposal distribution $q(\mathbf{x}[k] \mid \mathbf{x}[k-1], \mathbf{y}[k])$ is equal to the Markov transition $p(\mathbf{x}[k] \mid \mathbf{x}[k-1])$, which leads to the very


Fig. 8. Estimated positions of a human walking along a known path using a Gaussian model. The filter diverges and is unable to track the location of a person using a Guassian model.

simple weight update step. While this assumption makes for easy implementation, the efficiency of the particle filter is drastically reduced, since the current measurement is not used to propose new particle positions. The development and application of more efficient DFL particle filter designs is a topic for future research.

\subsection{Overview of Tracking Experiments and Results}

This section presents the results of tracking experiments utilizing the fade-level skew-Laplace likelihood models and particle filtering. We use the same experiment data as described in Section 2.2, which includes two deployments in drastically different environments: a bookstore and a through-wall house. For each experiment, we use the same parameters presented in Table 1 . The fact that tracking is effective in both environments with no changes in parameters is a testament to the robustness of the skewLaplace model.

We tested all of our tracking experiments using a fadelevel Gaussian model to show the necessity of the fade-level skew-Laplace model. In a process equivalent to that discussed in Section 2, we determined the mean and variance of the changes in RSS for each fade level and LOS position. We then tested the tracking using a Gaussian distribution. The Gaussian model was completely unusable, as it was not able to converge or track in any of our experiments. In fact, it seemed to have a diverging effect where particles wanted to "push away" from the true location of the person. The poor results for the Gaussian model are evident in Fig. 8.

In all experiments presented in the following sections, a calibration of RSS was taken while each surveillance area was vacant. The calibration stage lasted for approximately 30 seconds. The calibration RSS measurements are averaged to determine $\overline{\mathbf{z}}$ and the fade-level vector $\mathbf{F}$, as shown in Fig. 1. Additionally, particles are constrained to only the tracking area inside the area of interest. There is no use in proposing particles beyond the area of interest, as it will lead to wasted computational resources and additional error. 


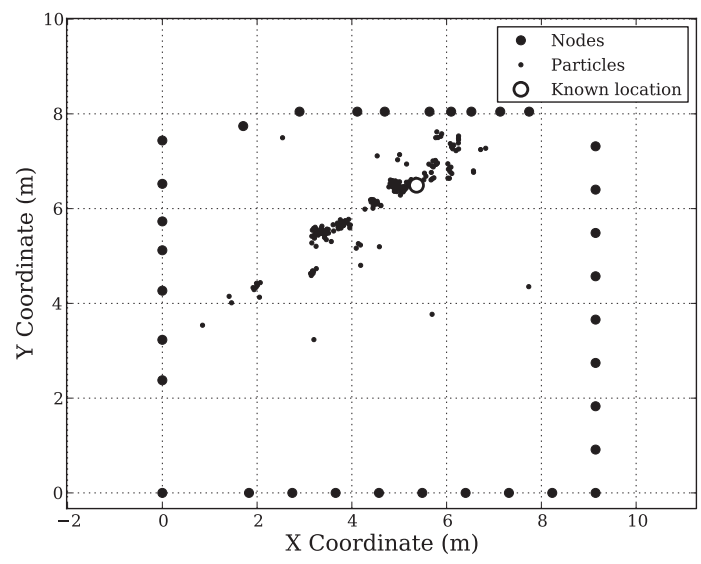

(a) Particle positions after 150 iterations.

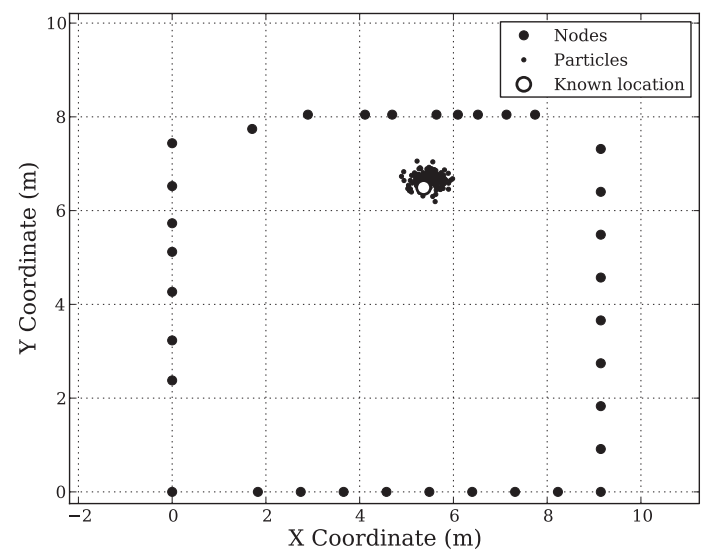

(b) Particle positions after 300 iterations.

Fig. 9. The convergence of the particle filter over time. After 150 iterations (a), the filter has determined that the person is located along a particular LOS path. After 300 iterations (b), the filter has completely converged around the location of the person.

\subsection{Results for Stationary Person Tracking through Walls}

A key benefit of the proposed models and algorithms is the ability to locate stationary people behind walls. VRTI tracking systems [2] are unable to locate stationary people, since the algorithms rely on the variance caused by motion. Here, the particle filter is able to locate stationary people as long as calibration means are available.

The convergence of the particle filter around a person's location is illustrated in Fig. 9. We define an iteration of the particle filter as the processing of a single link measurement. After 150 iterations of the filter, particles along a particular narrow area survive, while other areas are eliminated. This is because a particular link is reporting a statistically significant change in RSS, and the particle filter narrows its search to areas near that particular LOS. In this case, after 300 iterations, the particle filter has completely converged around the person's position.

To determine the accuracy of the statistical method for tracking stationary objects through walls, 20 trials were



Fig. 10. Estimated positions of a stationary person at different positions. The estimated position was taken after 3,000 iterations (approximately 3 seconds in time) of a particle filter with 200 particles. The average error for this experiment was 0.83 meters. performed. In each trial, a human stood completely motionless at a different known location on the interior of the surveillance area. The known and estimated positions are shown in Fig. 10. The average error over the 20 trials was 0.83 meters.

\subsection{Results for Tracking Moving People}

To test the accuracy of our model for tracking moving people, a human moves at a typical walking pace (approximately one meter per second) along a defined path in each of the deployments. The person's actual location is determined as described in Section 2.2, then compared with the particle filter estimation.

The fade-level skew-Laplace model and particle filter accurately tracks the location of a moving person in the bookstore environment. The average tracking error for the bookstore deployment was 0.58 and 0.9 meters for the through-wall experiment. Both of these results were produced using a filter with 100 particles. Example of typical tracking paths are provided in Figs. 11 and 12.

The cumulative error distribution functions for both experiments are shown in Fig. 13. The results show that
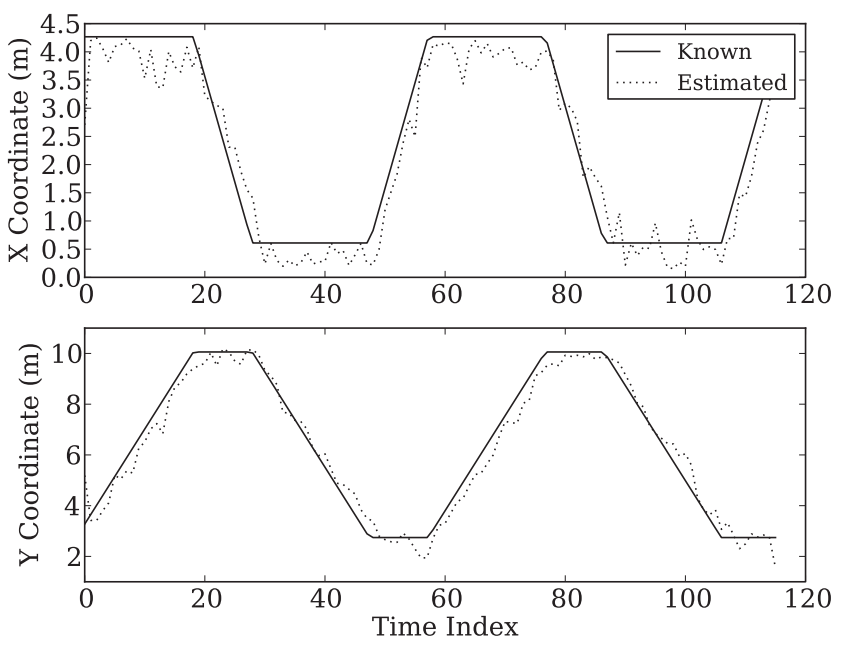

Fig. 11. Estimated positions of a human walking along a known path in a bookstore. Here, the particle filter uses 100 particles and tracks with an average error of 0.58 meters. 

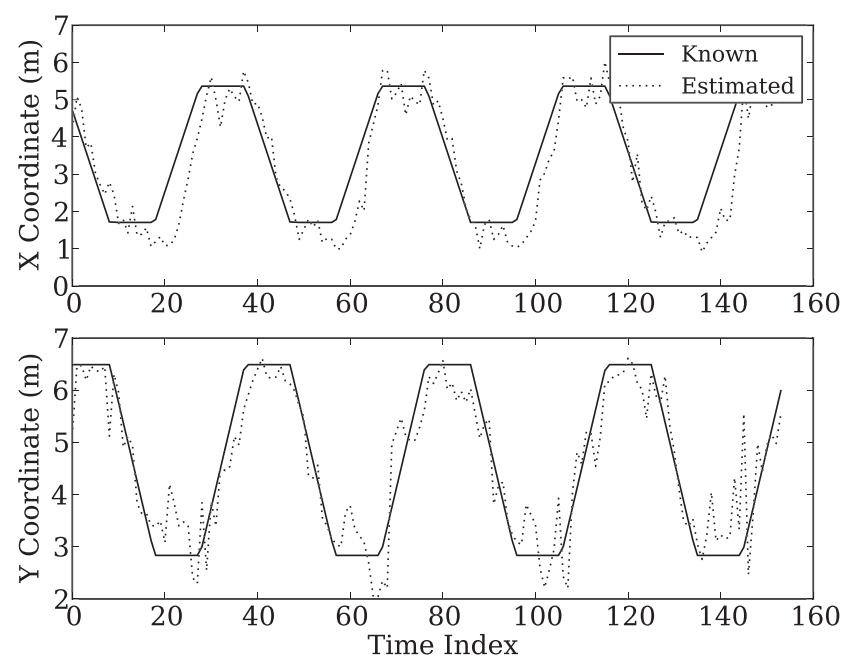

Fig. 12. Estimated positions of a human walking along a known path within the walls of a home. Here, the particle filter uses 100 particles and tracks with an average error of 0.9 meters.

with 100 particles, 90 percent of the tracking estimates are accurate to less than 0.9 meters for the bookstore and less than 1.6 meters for the through-wall experiment.

\subsection{Results for Tracking Two People}

Tracking multiple people is possible with the skew-Laplace model. In our algorithms, we do not modify the parameters provided in Table 1 even if multiple targets exist on the same LOS path of a link simultaneously. While this is a simplifying assumption, the model in its current form is capable of tracking more than one person. We should note that we did not test the limits of how many targets can be tracked using our model. Future work will extend the model to explicitly handle multiple targets in various configurations to obtain higher accuracy and quantify the limits of the associated model.

One challenge with tracking multiple targets is the association of trajectories to each person. When two or more people are moving close together, the particle filter

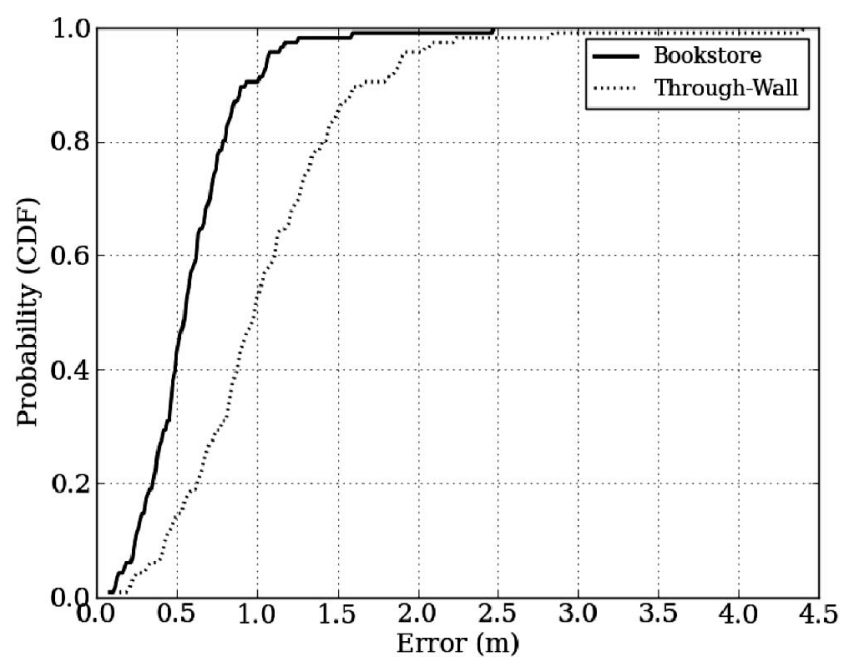

Fig. 13. The cumulative error distribution for tracking a moving person in both the bookstore and through-wall experiments. Here, 100 particles were used in the filter.

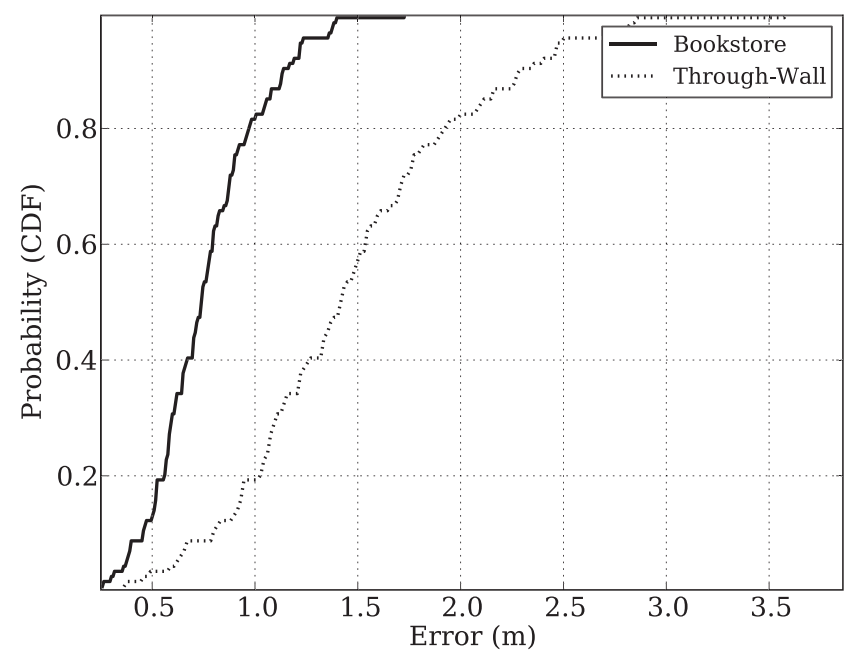

Fig. 14. The cumulative error distribution for tracking two people in both the bookstore and through-wall experiments. Here, 200 particles were used in the filter in both cases.

may confuse each trajectory and swap them. This challenge is present in all tracking algorithms, and can be addressed using more accurate methods and assumptions regarding the motion dynamics of the state space. Since this paper is more concerned with the model itself, we do not present any new techniques for addressing this challenge. For our error analysis, we use the global minimum distance estimate for each person to avoid errors that may occur due to trajectory swapping.

In the bookstore environment, the system was able to track two moving people with an average error of 0.84 meters with 200 particles. For the through-wall experiment, the average error was 1.1 meters using the same number of particles. Since the state space increases dramatically with more targets, more particles are required to maintain approximately the same average error. The tracking error CDFs for both environments is shown in Fig. 14. Plots of the particle clouds with their accompanying target locations are provided for each environment in Fig. 15.

\section{Related Work}

Various sensor technologies can be used for the purposes of DFL [1]. The most common form of a DFL sensor is the optical camera. Infrared and thermal cameras are also increasingly common in military and security applications. While these technologies are certainly valuable, visible light cameras depend on an external source of light. Furthermore, optical, thermal, and infrared sensors are hindered by opaque or insulating obstructions.

There is an advantage to using radio frequency sensors to infer people's locations instead of optical, thermal, and infrared sensors. RF waves have the ability to penetrate obstructions like walls, trees, and smoke. Thus, DFL systems that use RF sensors (RF-DFL) are capable of locating people through walls, in poor-sight outdoor environments, or in a smoke-filled buildings. These capabilities have obvious value for military organizations, police forces, and firefighter and rescue operations. Furthermore, RF-DFL systems often do not have the ability 


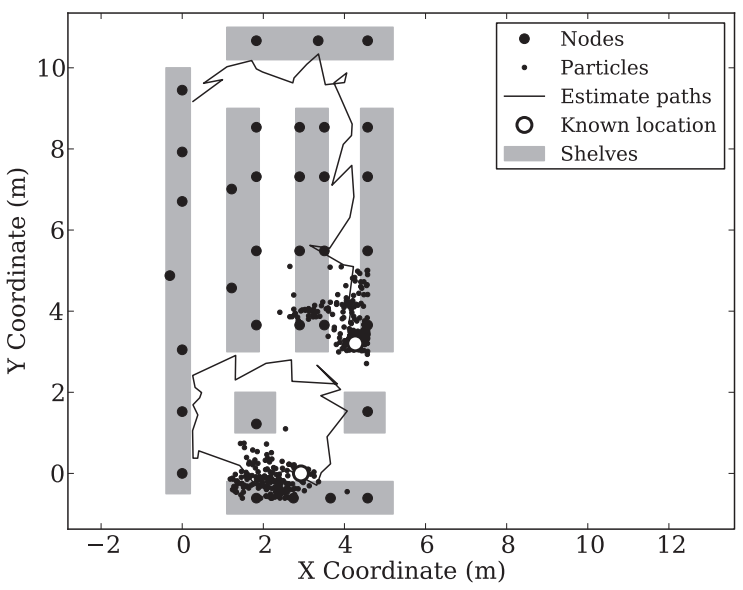

(a) Bookstore environment.

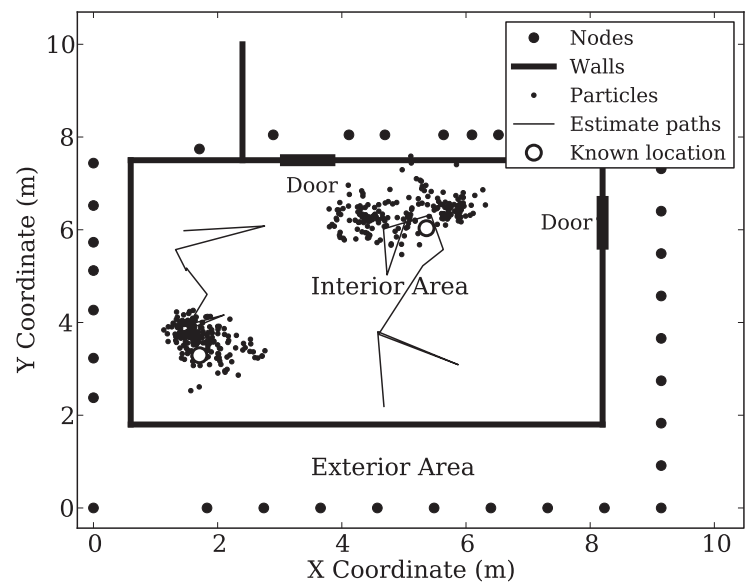

(b) Through-wall environment.

Fig. 15. Plots showing particle clouds and a history of tracking paths for two people moving within the network. The true paths were: (a) one person traveled from $(1.0,9.0)$ to $(4.0,9.0)$ to $(4.0,3.0)$, and the second person traveled from $(3.0,0.0)$ to $(3.0,2.5)$ to $(0.5,2.5)$ to $(0.5,0)$ to the original starting point; (b) one person traveled from $(5.8,3.0)$ to $(5.8,7.1)$, and the second person traveled from $(1.8,3.0)$ to $(1.8,7.1)$.

to identify people or get detailed information about the people's actions. In some applications this may be a limitation, but in others the additional preservation of privacy compared to camera surveillance may be desirable. The most common and widely used form of RF-DFL is ultrawideband (UWB) radar [17], [18]. UWB systems work by producing a very fast pulse of RF energy and recording the amplitudes, time delays, and phases of the reflections caused by objects and people in the vicinity. Some UWB systems are monostatic, meaning the transmitter and receiver are incorporated into a single device. Others are multistatic, where a single pulse transmission may be received by multiple devices deployed throughout an area.

Recently, researchers have begun to study and develop DFL systems that use the received signal strength (RSSDFL) of links in narrowband RF sensor networks. The advantage to this approach lies in the fact that RF sensors capable of measuring signal strengths are ubiquitous and inexpensive. The cost of each node is orders of magnitude lower than a UWB device, so deploying a network with tens or hundreds of nodes is financially feasible in many applications. Furthermore, RSS measurements can be obtained from off-the-shelf devices like wireless routers, access points, wireless sensor modules, and cell phones.

One approach to RSS-DFL in wireless networks is to use RSS fingerprinting, or radio maps [3]. In this approach, the system is trained by a person standing at many predefined positions, and RSS measurements are recorded while the person stands at each location. When the system is in use, RSS measurements are compared with the training data, and the closest matching position is selected. The accuracy can be further refined by combining multiple best matching positions and using an appropriate interpolation [4].

The strength of the RSS fingerprinting approach is that the variations in RSS caused by the person in the multipath environment are an advantage. Each human position will lead to very different vector of RSS measurements, making it easier to detect the location. The weakness of such a system is the need for manual training and maintenance. Measurements must be taken offline, and changes to the environment such as doors opening or moved furniture will corrupt the training. Furthermore, the training becomes exponentially difficult for localization of multiple people.

Zhang et al. [5] present an algorithm which directly estimates a human's position from RSS measurements. In this work, when a link measures RSS variation above a threshold, it is assumed that the person is located within a rectangle centered at the midpoint of the line between the transmitter and receiver. A "best cover algorithm" then estimates the person's position, which is input into a tracking filter. This work was extended in [6] to use a clustering algorithm for multiobject tracking.

One approach to DFL is to estimate an image of the change in environment. This image can then be used to infer the motion and activity within the environment, either by a human operator, or by an image processing algorithm. Image estimation from measurements along different spatial filters through a medium is generally referred to as tomographic image reconstruction. For RF sensors, this is termed radio tomographic imaging (RTI) [19], [9], [20], [2]. In [9], the attenuation in $\mathrm{dB}$ caused by each voxel in the environment is imaged using measurements of RSS for each link in a dense wireless network. This technique can be referred to as shadowing-based RTI, since the measurements effectively measure shadowing loss, and the image estimates are shown to accurately display the location one or two people in the deployment area [9]. The linear model for shadowing loss is based on correlated shadowing models [21], [19].

Another modality of RTI is termed variance-based RTI, in which the windowed variance of RSS on each link is used as the measurement, and the estimated image represents a quantification of the motion within each voxel. Experimental tests reported in [2] show that variance-based RTI can image the motion occurring inside a house, when sensors are placed only outside of its external walls. In the case of imaging motion through building walls, we can have the problem that the multipath which travel around the building can be stronger than the power in paths which traveled through the building. Analytical results in [2] 
suggest that the change in variance can be detected even when the power in the affected multipath is $10 \mathrm{~dB}$ less than the multipath which do not go through the building.

\section{CONCLUSION}

Previous work in the field of RSS-based DFL shows that it is possible to locate humans using only RSS measurements, even through walls. In particular, RTI provides a method for RSS-DFL that does not require exhaustive training information. Previous work in model-based RSS-DFL has been unable to locate stationary or slowly moving people in highly obstructed areas. This paper provides a statistical model and inversion method that can be applied to locate stationary as well as moving people. It can also be applied to track multiple people behind walls and in complex indoor environments, an extension that has not been presented in previous work.

The amount of fading on a static link is an important factor in determining the distribution of RSS when a person enters the area near the link. If the link is already in a deep fade, the disturbance a person causes to the multipath will tend to increase the RSS. Links in deep fades also exhibit more variance, since even slight changes to multipath components can bring the link out of the fade. Links that experience antifades, however, exhibit the opposite behavior. Changes to the environment due to human presence tend to bring signal power down, and variances are significantly lower.

The skew-Laplace distribution is a reasonable representation for the temporal changes in RSS measurements. The mode and decay parameters of the distribution are dependent on the fade level of the link as well as the person's position. When a person is on the LOS path of the link, RSS fluctuations are significantly larger than when the person is away from the LOS. Each parameter, for both the LOS and off-LOS cases, is seen to be linear with the fade level.

Experiments using a particle filter and the fade-level skew-Laplace likelihood models demonstrate the method's effectiveness in locating stationary and moving people behind walls. Previous work in through-wall VRTI in wireless networks was unable to locate stationary objects. The model is also robust to multiple people moving. Our experiments show that multiple moving people can be tracked without modification to the model or its parameters.

Multiple opportunities for improvement exist. First, other methods, besides the fade-level skew-Laplace model, certainly exist, some of which may provide a better statistical divergence for varying human and object positions. A model that has higher contrast for the on-LOS distributions versus the off-LOS distributions will lead to more accurate DFL tracking. Next, improved particle filtering methods will likely result in more accurate tracking. Finally, a model that explicitly accounts for the effects of multiple people needs to be investigated. As many people enter the area near a wireless link, interactions of the people with the multipath components become more complex. The RSS measurement statistics of such a scenario need to be modeled, quantified, and then applied in an estimation framework like the particle filter.

\section{ACKNOWLEDGMENTS}

This material is based upon work supported by the US National Science Foundation under Grant Nos. 0748206 and 1035565.

\section{REFERENCES}

[1] N. Patwari and J. Wilson, "RF Sensor Networks for Device-Free Localization and Tracking," Proc. IEEE, 2010.

[2] J. Wilson and N. Patwari, "See through Walls: Motion Tracking Using Variance-Based Radio Tomography Networks," IEEE J. Selected Areas in Comm., 2010.

[3] M. Youssef, M. Mah, and A. Agrawala, "Challenges: Device-Free Passive Localization for Wireless Environments," Proc. ACM MobiCom, 2007.

[4] M. Seifeldin and M. Youssef, "Nuzzer: A Large-Scale Device-Free Passive Localization System for Wireless Environments," technical report, 2009.

[5] D. Zhang, J. Ma, Q. Chen, and L. Ni, "An RF-Based System for Tracking Transceiver-Free Objects," Proc. IEEE Fifth Ann. Int'l Conf. Pervasive Computing and Comm., 2007.

[6] D. Zhang and L. Ni, "Dynamic Clustering for Tracking Multiple Transceiver-Free Objects," Proc. IEEE Int'l Conf. Pervasive Computing and Comm., pp. 1-8, 2009.

[7] J. Wilson and N. Patwari, "Spin: A Token Ring Protocol for RSS Collection," http://span.ece.utah.edu/spin, 2011.

[8] N. Patwari and J. Wilson, "People-Sensing Spatial Characteristics of RF Sensor Networks," Technical Report ArXiv: 0911.1972v1, SPAN Lab, Univ. of Utah, Oct. 2009.

[9] J. Wilson and N. Patwari, "Radio Tomographic Imaging with Wireless Networks," IEEE Trans. Mobile Computing, vol. 9, no. 5, pp. 621-632, Jan. 2010.

[10] P. Hande, J. Smith, and D. Reed, "An Analysis of Fading Mechanisms for Fixed Antennas," Proc. IEEE Vehicular Technology Conf., 2000.

[11] T.S. Rappaport, Wireless Communications: Principles and Practice. Prentice Hall, 2002.

[12] B. Ristic, S. Arulampalam, and N. Gordon, Beyond the Kalman Filter: Particle Filters for Tracking Applications. Artech House, 2004.

[13] M.K. Pitt and N. Shepard, "Filtering via Simulation: Auxiliary Particle Filters," I. Am. Statistical Assoc., vol. 94, pp. 590-599, 1999.

[14] R. van der Merwe, A. Doucet, N. de Freitas, and E. Wan, "The Unscented Particle Filter," technical report, Eng. Dept., Cambridge Univ., 2000.

[15] S. Sarkka, A. Vehtari, and J. Lampinen, "Rao-Blackwellized Particle Filter for Multiple Target Tracking," Information Fusion, vol. 8, no. 1, pp. 2-15, 2007.

[16] C. Robert and G. Casella, Monte Carlo Statistical Methods. Springer, 2004.

[17] A.R. Hunt, "Image Formation through Walls Using a Distributed Radar Sensor Network," Proc. SPIE Conf. Sensors, and Command, Control, Comm., and Intelligence (C3I) Technologies for Homeland Security and Homeland Defense IV, vol. 5778, pp. 169-174, May 2005.

[18] Camero Tech, http://camero-tech.com, 2011.

[19] N. Patwari and P. Agrawal, "Effects of Correlated Shadowing: Connectivity, Localization, and RF Tomography," Proc. IEEE/ ACM Int'l Conf. Information Processing in Sensor Networks (IPSN '08), Apr. 2008.

[20] M.A. Kanso and M.G. Rabbat, "Compressed RF Tomography for Wireless Sensor Networks: Centralized and Decentralized Approaches," Proc. Distributed Computing in Sensor Systems (DCOSS), 2009.

[21] P. Agrawal and N. Patwari, "Correlated Link Shadow Fading in Multi-Hop Wireless Networks," IEEE Trans. Wireless Comm., vol. 8, no. 8, pp. 4024-4036, Aug. 2009. 


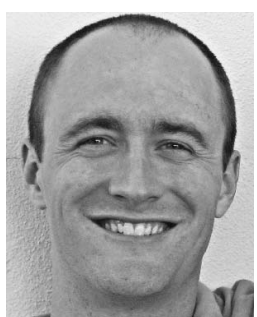

Joey Wilson received the $\mathrm{BS}, \mathrm{ME}$, and $\mathrm{PhD}$ degrees from the Department of Electrical and Computer Engineering, University of Utah. He is the founder and president of Xandem Technology, a company dedicated to radio tomography and wireless network device-free sensing technologies. His research interests include signal processing, estimation and detection theory, and communications. Prior to his doctorate studies, he worked in research and development at L-3 Communications (Communications Systems West) in Salt Lake City. Outside of engineering, he enjoys his family, the guitar, ultimate frisbee, climbing, and the outdoors.



Neal Patwari received the BS and MS degrees from Virginia Tech in 1997 and 1999, respectively, and the PhD degree from the University of Michigan, Ann Arbor, in 2005, all in electrical engineering. He was a research engineer at Motorola Labs, Florida, between 1999 and 2001. Since 2006, he has been at the University of Utah, where he is an assistant professor in the Department of Electrical and Computer Engineering, with an adjunct appointment in the School of Computing. He directs the Sensing and Processing Across Networks (SPAN) Lab, which performs research at the intersection of statistical signal processing and wireless networking. He has served on technical program committees for IEEE conferences SECON, ICDCS, DCOSS, ICCCN, ICC, and MILCOM, and was the publicity chair for IPSN 2010. He is an associate editor of the IEEE Transactions on Mobile Computing. His research interests include radio channel signal processing, in which radio channel measurements are used for purposes of security, localization, and networking.

$\triangleright$ For more information on this or any other computing topic, please visit our Digital Library at www.computer.org/publications/dlib. 